\title{
Long-term quality of life and psycho-social outcomes after oropharyngeal cancer surgery and radial forearm free-flap reconstruction: A GETTEC prospective multicentric study
}

\author{
Alexandre Bozec ${ }^{\text {a, }}$, Pierre Demez ${ }^{\mathrm{b}}$, Jocelyn Gal ${ }^{\mathrm{c}}$, Emmanuel Chamorey ${ }^{\mathrm{c}}$, \\ Esteban Brenet ${ }^{\mathrm{e}}$, Olivier Dassonville ${ }^{\mathrm{a}}$, Gilles Poissonnet ${ }^{\mathrm{a}}$, José Santini ${ }^{\mathrm{a}}$, \\ Emmanuel Babin ${ }^{j}$ \\ a Institut Universitaire de la Face et du Cou, Centre Antoine Lacassagne, Université Côte d'Azur, Nice, France \\ ${ }^{\mathrm{b}}$ Department of Otorhinolaryngology and Head and Neck Surgery, University Hospital of Liège, Belgium \\ ' Department of Statistics, Centre Antoine Lacassagne, Université Côte d'Azur, Nice, France \\ ${ }^{\mathrm{d}}$ Department of Maxillo-facial and Head and Neck Surgery, Centre François-Baclesse, Caen, France \\ e Department of Otorhinolaryngology and Head and Neck Surgery, University Hospital of Reims, France \\ ${ }^{\mathrm{f}}$ Department of Medical Oncology, Centre Antoine Lacassagne, Université Côte d'Azur, Nice, France \\ ${ }^{g}$ Department of Radiotherapy, Centre Antoine Lacassagne, Université Côte d'Azur, Nice, France \\ ${ }^{\mathrm{h}}$ Department of Otorhinolaryngology and Head and Neck Surgery, Institut Curie, Paris, France \\ i Department of Otorhinolaryngology and Head and Neck Surgery, Hospital of Annecy, France \\ ${ }^{j}$ Department of Otorhinolaryngology and Head and Neck Surgery, University Hospital of Caen, France
} Marie-Yolande Louis ${ }^{\mathrm{d}}$, David Blanchard ${ }^{\mathrm{d}}$, Dominique De Raucourt ${ }^{\mathrm{d}}$, Jean-Claude Merol ${ }^{\mathrm{e}}$, Frédéric Peyrade ${ }^{\mathrm{f}}$, Karen Benezery ${ }^{\mathrm{g}}$, Maria Lesnik ${ }^{\mathrm{h}}$, Etienne Berta ${ }^{\mathrm{i}}$, Pierre Ransy ${ }^{\mathrm{b}}$,

\section{A R T I C L E I N F O}

\section{Article history:}

Received 19 July 2017

Received in revised form

25 October 2017

Accepted 22 November 2017

\section{Keywords:}

Quality of life

Psychosocial outcomes

Anxiety

Depression

Head and neck cancer

Surgery

Oropharynx cancer

\begin{abstract}
A B S T R A C T
Objective: To assess long-term quality of life (QoL) and psycho-social outcomes, and to determine their predictive factors after oropharyngeal cancer (OPC) surgery and radial forearm free-flap (RFFF) reconstruction.

Methods: Patients who had undergone OPC surgery and RFFF reconstruction who were still alive and disease-free at least 1 year after surgery were enrolled in this prospective multicentric study. Patients completed the European Organization for Research and Treatment of Cancer (EORTC) Core (QLQ-C30) and Head and Neck Cancer (QLQ-H\&N35) QoL questionnaires, the Voice Handicap Index (VHI-10) questionnaire and the Hospital Anxiety and Depression Scale (HADS). The level of dysphagia was evaluated using the Dysphagia Handicap Index (DHI) and the Dysphagia Outcomes and Severity Scale (DOSS). Predictive factors of these clinical outcomes were determined in univariate and multivariate analysis. Results: A total of 58 patients were included in this study. Long-term QoL and functioning scales scores were well-preserved (all superior to 70\%). Main persistent symptoms were fatigue, reduced sexuality and oral function-related disorders (swallowing, teeth, salivary and mouth-opening problems). HADS anxiety and depression scores were 7.2 and 5.4, respectively. Twenty-one (36\%) patients presented an anxiodepressive disorder (HADS global score $\geq 15$ ). Among the 21 patients who were still working before surgery, $11(52 \%)$ had returned to work at the time of our study. The HADS global score ( $<<0.001$ ) was the main predictor of QoL, VHI-10 and DOSS scores.

Conclusions: Psychological distress is the main determinant of long-term QoL and is therefore of critical importance in the multidisciplinary management of OPC patients.
\end{abstract}

๑) 2017 Elsevier Ltd. All rights reserved.
* Corresponding author. Institut Universitaire de la Face et du Cou, 31 avenue de Valombrose, 06103 Nice, France.

E-mail address: alexandre.bozec@nice.unicancer.fr (A. Bozec).

\section{Introduction}

The role of surgery in patients with oropharyngeal cancer (OPC) is controversial and has recently declined with the increasing 
proportion of OPC associated with HPV (human papillomavirus) $[1,2]$. Nevertheless, surgery still plays an important role in OPC treatment, either as a salvage procedure or as a primary treatment for selected patients [1]. Over the past three decades, microvascular free tissue transfer has become the gold standard in reconstruction of large head and neck oncologic defects $[3,4]$. For most authors, the fasciocutaneous radial forearm free flap (RFFF) is recognized to be the method of choice for reconstruction of oropharyngeal soft tissue defects $[3,4]$. The reliability of head and neck free flap reconstruction is now well-established and recent studies have investigated the functional outcome of patients undergoing this type of oncologic surgery [3-5]. Nowadays, quality of life (QoL) is considered to be a crucial issue in the evaluation of any cancer therapy and is therefore the endpoint in the evaluation of this complex surgery.

Although several studies have investigated QoL after OPC treatment, particularly after chemoradiation therapy, few authors have specifically explored long-term QoL in patients undergoing oncologic surgery with RFFF reconstruction [5,6]. Moreover, in this type of patient, psychosocial outcomes and their possible impact on QoL, as well as other potential predictors of QoL, have been insufficiently explored [7].

The aims of this international multicentric prospective study were to assess long-term QoL and psychosocial outcomes, and to determine their predictive factors after OPC surgery and RFFF reconstruction.

\section{Material and methods}

This study was conducted by the GETTEC (Groupe d'Etude des Tumeurs de la Tête et du Cou) study group.

\subsection{Patients}

The inclusion criteria of our study were as follows:

- Oncologic surgery and RFFF reconstruction for primary or recurrent oropharyngeal squamous cell carcinoma, between January 2002 and August 2015, at four tertiary cancer care centers (France: Nice, Caen and Reims; Belgium: Liège)

- Alive and disease-free patients at the time of our study (November 2016, i.e. at least 1 year after surgery)

The exclusion criteria were as follows:

- Patients who were not fluent in French or who could not answer the EORTC questionnaires for physical, psychological, or other reasons

- Unsuccessful microvascular reconstruction (total free flap necrosis)

- Intercurrent disease (any severe cardiac, respiratory, hepatic, renal or neurological disease) responsible for a potential significant impact on patient daily life and QoL.

Patients were staged according to the 2009 American Joint Committee on Cancer (AJCC) staging system.

\subsection{Ethical consideration}

The protocol and all accompanying material provided to patients were reviewed and approved by institutional ethics committees prior to the start of the study. The study was conducted in compliance with the Helsinki Declaration. Informed consent was obtained from each of the participants.

\subsection{QoL evaluation}

Patients completed the French versions of the European Organization for Research and Treatment of Cancer (EORTC) Core QoL Questionnaire (QLQ-C30) and the EORTC Head and Neck Cancer QoL Questionnaire (QLQ-H\&N35) before therapy and at least 1 year after surgery (November 2016). As recommended by the EORTC, the scales and single item variables of the QLQ-C30 and H\&N35 questionnaires were transformed linearly into a score from 0 to 100 . A high score for a functioning scale and for the global QoL scale represents a better level of functioning, whereas a high score for a symptom scale or a single-item scale denotes a high level of symptoms or problems.

\subsection{Functional evaluation}

Speech and swallowing outcomes were determined before therapy and more than 1 year after surgery (November 2016) as follows:

- Patients completed the Voice Handicap Index 10 (VHI-10) selfquestionnaire. This well-validated and widely-used questionnaire comprises 10 questions. A total score ranging between 0 and 40 is obtained for each patient (higher scores reflect additional speech problems)

- Patients completed the Dysphagia Handicap Index (DHI) selfquestionnaire. The $\mathrm{DHI}$ is a 30 -item questionnaire on deglutition-related aspects of daily life (5-point rating scale: $0-4)$. The minimum scores range from zero (indicating no handicap) to 120 (indicating maximum handicap).

- Swallowing was evaluated by the same head and neck surgeon at each participating institution using the Dysphagia Outcome and Severity Scale (DOSS). This well-standardized 7-step scale is used to classify the ability to swallow, level 7 corresponding to normal swallowing.

We also analyzed patient responses to the open question: "What are the main physical changes since OPC surgery that impact your daily life?"

\subsection{Psychosocial outcomes}

Patients completed the French version of the Hospital Anxiety and Depression Scale (HADS) questionnaire before therapy and at least 1 year after surgery (November 2016). The HADS is widely used to measure anxiety and depression, and is considered an effective screening tool to assess psychological distress in cancer patients. It consists of two subscales (HADS-A and HADS-D) that evaluate anxiety and depression, respectively. Scores for each subscale range from 0 to 21 and a cut-off score of 11 is considered to be a good indicator of anxiety or depressive disorders. For the global score, a cut-off score of 15 is recognized to provide a good indicator of anxio-depressive disorders and psychological distress.

To measure more accurately the impact of treatment on social life, we also investigated the evolution of the patient's professional status after therapy (qualitative and/or quantitative changes in professional activities, duration of sick leave).

We also analyzed patient responses to 2 open questions: "What are the main changes in your family life since OPC surgery?" and: "What are the main changes in your social life since OPC surgery?"

\subsection{Statistical analyses}

We analyzed the influence on QLQ-C30, QLQ-H\&N35, VHI, DOSS and HADS scores of the following factors: age $(<v s>65$ years), 
gender, education level ( $<v s \geq$ high school diploma), professional activity (yes $v s$ no), alcohol consumption (before and after surgery), tobacco consumption (before and after surgery), T-stage ( $<v s \geq 3$ ), $\mathrm{N}$-stage $(<v s \geq 1)$, tumor recurrence (yes: salvage surgery $v s$ no: primary surgical treatment), anatomic subsites of the tumor (base of tongue, lateral pharyngeal wall, soft palate, posterior pharyngeal wall), type of surgical approach (transmandibular or transoral), adjuvant treatment (postoperative radiotherapy \pm chemotherapy: $\mathrm{RT} \pm \mathrm{CT}$ : yes vs no) and HADS scores.

Univariate analyses were performed using Student's t tests, Wilcoxon tests, Pearson's correlation tests or Spearman's correlation tests, when appropriate. For multivariate analysis (conducted only when more than one factor was significant in univariate analyses), all variables associated with $\mathrm{p}<0.10$ on univariate analysis were included in linear regression models with forward stepwise selection.

As previously reported [8], several scales and single-item variables were analyzed together to facilitate presentation of the QoL scores:

- fatigue, nausea and vomiting, pain, dyspnea, insomnia, appetite loss, constipation and diarrhea were grouped together to obtain the mean score for general symptoms

- social contact, social eating, pain, swallowing, senses, speech, sex-life, teeth, open mouth, dry mouth, sticky saliva, cough and feeling ill were grouped together to obtain the mean score for head and neck symptoms.

All statistical analyses were performed at 5\% alpha risk or $95 \%$ confidence interval by the biostatistician using R.3.0.1 software on Windows.

\section{Results}

\subsection{Patients' clinical characteristics}

A total of 60 patients were primarily enrolled in the study. Two patients were secondarily excluded from the study because of a severe intercurrent disease ( 2 cases of definitive hemiplegia due to cerebral stroke) occurring before the post-therapeutic evaluation period. Finally, 58 patients were included in the study (41 males and 17 females, mean age $61.5 \pm 11.1$ years, range $27-80$ years). Their main clinical characteristics are shown in Table 1 . The mean time between surgery and QoL assessment was $4.5 \pm 3.3$ years.

\subsection{EORTC QLQ-C30 and QLQ-H\&N35 scores}

The EORTC QLQ-C30 and QLQ-H\&N35 scores obtained by patients are shown in Tables 2 and 3.

\subsection{Patients' functional and psychosocial outcomes}

Patients' functional and psychosocial outcomes are presented in Table 4.

Patient responses to the open question: "What are the main physical changes since OPC surgery that impact your daily life?" are presented in Fig. 1.

Among the 16 patients who were heavy drinkers (average of more than two drinks per day) at the time of surgery, 10 (63\%) had stopped alcohol consumption at the time of the study. Among the 26 patients who were regular smokers at the time of surgery, 19 (73\%) had stopped tobacco consumption at the time of the study.

Twenty-one (36\%) of the 58 patients included in our study were still working before surgery. At the time of the study, 11 (52\%) patients had resumed a professional activity but 6 of them had required changes at work ( 3 shorter working hours and 3 qualitative changes). Among the 10 patients who did not return to work, 6 were over 55 years. Mean duration of sick leave for patients who had resumed professional activity was $13.8 \pm 9.2$ months.

Between the pre- and post-therapeutic periods, there was no evolution regarding patients' marital status. Fifty patients (86\%) did not report any change in their family life, 5 patients reported having sex less frequently, and 3 patients reported feeling closer to and being more confident with their partner. Forty-three patients (74\%) reported no change in their social life, 9 patients reported having fewer social contacts, and 6 patients reported eating out less often.

\subsection{Predictive factors of clinical and psychological outcomes after treatment}

Predictive factors of QoL scores and of functional and psychological outcomes after treatment, in univariate and multivariate analysis, are shown in Table 5.

\section{Discussion}

In this prospective multicentric international study on OPC patients undergoing oncologic surgery and RFFF reconstruction with or without postoperative $\mathrm{RT} \pm \mathrm{CT}$, we showed that, long-term after therapy, global QoL and functioning scales were well-preserved. Interestingly, in a QoL study on patients with oral/oropharyngeal cancer treated by surgery with or without RT, de Graeff et al. found

Table 1

Clinical characteristics of the 58 patients.

\begin{tabular}{|c|c|c|}
\hline Clinical characteristics & All patients $(n=58)$ & Percentage (\%) \\
\hline Gender: men/women & $41 / 17$ & $71 / 29$ \\
\hline Age: $<70 />70$ years & $47 / 11$ & $81 / 19$ \\
\hline Educational level: $\leq \mid>$ high school diploma & $40 / 18$ & $69 / 31$ \\
\hline Professional activity: current employed/unemployment-invalidity/retired & $21 / 12 / 25$ & $36 / 21 / 43$ \\
\hline Current smoker at the time of surgery: yes/no & $26 / 32$ & $45 / 55$ \\
\hline Heavy drinker at the time of surgery: yes/no & $16 / 42$ & $28 / 72$ \\
\hline T-Stage: T2/T3/T4 & $30 / 17 / 11$ & $52 / 29 / 19$ \\
\hline N-Stage: $\mathrm{N} 0 / \mathrm{N} 1 / \mathrm{N} 2 \mathrm{a}-\mathrm{C}$ & $26 / 10 / 22$ & $45 / 17 / 38$ \\
\hline Overall stage: II/III or IV & $15 / 43$ & $26 / 74$ \\
\hline Primary treatment/salvage surgery & $53 / 5$ & $91 / 9$ \\
\hline Tumor site: $\mathrm{BT} / \mathrm{LPW} / \mathrm{PPW} / \mathrm{SP}$ & $26 / 40 / 5 / 34$ & $44 / 69 / 8 / 59$ \\
\hline Surgical approach: transoral/transoral and transcervical/transmandibular & $12 / 37 / 9$ & $21 / 64 / 15$ \\
\hline Adjuvant treatment: $\mathrm{No} / \mathrm{RT} / \mathrm{RT}+\mathrm{CT}$ & $14 / 24 / 20$ & $24 / 41 / 35$ \\
\hline
\end{tabular}

Tumor site: BT: base of tongue, LPW: lateral pharyngeal wall, PPW: posterior pharyngeal wall, SP: soft palate (some tumors invaded more than 1 tumor site); RT: radiotherapy; CT: concurrent chemotherapy. 
Table 2

Results of the European organization for research and treatment of cancer core quality of life questionnaire (QLQ-C30).

\begin{tabular}{|c|c|c|c|c|c|c|}
\hline Variables & $\begin{array}{l}\text { Mean score } \\
\text { before therapy }\end{array}$ & $\begin{array}{l}\text { Mean score } \\
\text { after therapy }\end{array}$ & Standard deviation ${ }^{a}$ & Cronbach's Alpha ${ }^{a}$ & Lower limit CI 95\%a & Upper limit CI 95\% \\
\hline Global quality of life & 75.1 & 71.0 & 23.2 & 0.94 & 0.85 & 0.98 \\
\hline Physical functioning & 89.2 & 82.4 & 18.4 & 0.75 & 0.64 & 0.85 \\
\hline Role functioning & 88.4 & 75.8 & 27.6 & 0.88 & 0.69 & 0.95 \\
\hline Emotional functioning & 72.0 & 74.0 & 28.5 & 0.91 & 0.83 & 0.94 \\
\hline Cognitive functioning & 87.0 & 78.6 & 25.0 & 0.74 & 0.41 & 0.87 \\
\hline Social functioning & 92.1 & 76.9 & 31.7 & 0.93 & 0.82 & 0.97 \\
\hline Fatigue & 19.2 & 32.0 & 29.2 & 0.9 & 0.81 & 0.94 \\
\hline Nausea and vomiting & 2.1 & 5.6 & 13.6 & 0.74 & 0.16 & 0.98 \\
\hline Pain & 30.7 & 23.8 & 33.2 & 0.9 & 0.8 & 0.95 \\
\hline Dyspnea & 9.1 & 23.0 & 32.5 & NA & NA & NA \\
\hline Insomnia & 21.2 & 26.8 & 30.9 & NA & NA & NA \\
\hline Loss of appetite & 12.2 & 24.6 & 30.4 & NA & NA & NA \\
\hline Constipation & 7.6 & 19.1 & 27.7 & NA & NA & NA \\
\hline Diarrhea & 4.5 & 11.9 & 23.1 & NA & NA & NA \\
\hline Financial difficulties & 6.2 & 18.2 & 28.7 & NA & NA & NA \\
\hline
\end{tabular}

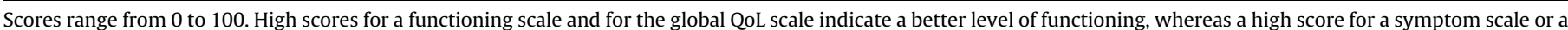

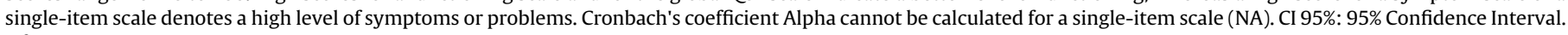

a Related to QoL scores after therapy.

Table 3

Results of the European organization for research and treatment of cancer head and neck cancer quality of life questionnaire (QLQ-H\&N35).

\begin{tabular}{|c|c|c|c|c|c|c|}
\hline Variable & $\begin{array}{l}\text { Mean score } \\
\text { before therapy }\end{array}$ & $\begin{array}{l}\text { Mean score } \\
\text { after therapy }\end{array}$ & Standard deviation $^{a}$ & Cronbach's Alpha $^{a}$ & Lower limit CI 95\% & Upper limit CI 95\% \\
\hline Pain & 33.2 & 26.0 & 30.2 & 0.9 & 0.81 & 0.95 \\
\hline Swallowing & 15.4 & 29.1 & 26.4 & 0.82 & 0.64 & 0.9 \\
\hline Sensory problems & 6.2 & 18.3 & 20.7 & 0.64 & 0.36 & 0.82 \\
\hline Speech problems & 5.3 & 25.5 & 24.9 & 0.59 & 0.42 & 0.7 \\
\hline Social eating problems & 12.6 & 33.7 & 31.2 & 0.86 & 0.73 & 0.93 \\
\hline Social contact problems & 7.5 & 14.1 & 19.5 & 0.8 & 0.59 & 0.91 \\
\hline Reduced sexuality & 12.7 & 43.1 & 41.7 & 0.98 & 0.96 & 1 \\
\hline Teeth & 14.7 & 37.4 & 40.3 & NA & NA & NA \\
\hline Mouth opening & 11.2 & 46.3 & 39.4 & NA & NA & NA \\
\hline Dry mouth & 10.2 & 54.5 & 33.9 & NA & NA & NA \\
\hline Sticky saliva & 11.4 & 47.2 & 35.7 & NA & NA & NA \\
\hline Coughing & 7.2 & 25.2 & 31.4 & NA & NA & NA \\
\hline Feeling ill & 6.1 & 12.2 & 20.8 & NA & NA & NA \\
\hline
\end{tabular}

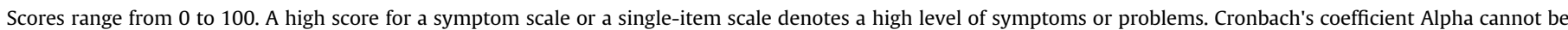
calculated for a single-item scale (NA). CI 95\%: 95\% Confidence Interval.

${ }^{a}$ Related to QoL scores after therapy.

Table 4

Patient functional and psychosocial outcomes.

\begin{tabular}{|c|c|c|c|c|}
\hline Functional and psychosocial outcomes & $\begin{array}{l}\text { Mean score (SD) } \\
\text { before therapy }\end{array}$ & $\begin{array}{l}\text { Mean score (SD) } \\
\text { after therapy }\end{array}$ & Number of Cases after therapy & $\begin{array}{l}\text { Percentage (\%) } \\
\text { after therapy }\end{array}$ \\
\hline VHI-10: $0-10 / 10-20 / 20-30 / 30-40$ & $4.2(2.1)$ & $12.8(10.6)$ & $25 / 20 / 8 / 5$ & $43 / 34 / 14 / 9$ \\
\hline DHI: $0-30 / 31-60 / 61-90 / 91-120$ & $10.1(7.3)$ & $37.5(27.4)$ & $27 / 15 / 15 / 1$ & $47 / 26 / 26 / 2$ \\
\hline DoSS: $1-2 / 3-5 / 6-7$ & $6.4(0.4)$ & $5.5(1.0)$ & $1 / 27 / 30$ & $2 / 46 / 52$ \\
\hline HADS - score A: 0-10/11-21 & $6.5(3.7)$ & $7.2(4.4)$ & $43 / 15$ & $74 / 26$ \\
\hline HADS - score D: 0-10/11-21 & $3.8(2.1)$ & $5.4(4.5)$ & $48 / 10$ & $83 / 17$ \\
\hline HADS - global score: $0-14 / 15-42$ & $10.3(5.1)$ & $12.6(8.4)$ & $37 / 21$ & $64 / 36$ \\
\hline
\end{tabular}

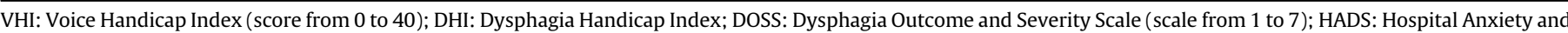
Depression Scale (score from 0 to 21 for anxiety [A] and depression [D] scores and from 0 to 42 for global score).

remarkably similar results with a score 1 year after treatment of nearly $70 \%$ for global QoL and nearly $80 \%$ for all functioning scales [9]. The present study was conducted at a median duration of 4.5 years after treatment. However, in disease-free patients, QoL at 1 year is generally recognized to be a good predictor of long-term QoL $[10,11]$. There was a low level of general symptoms except for fatigue, which was the main persistent general symptom. In this regard, in most QoL studies on head and neck cancer patients, including the above-mentioned de Graeff study, fatigue remains the main general symptom long-term after treatment, particularly in patients receiving $\mathrm{RT}+\mathrm{CT}[6,8,9]$.
In contrast, the impact of treatment on QoL scores was evident for most head and neck symptoms, including swallowing and speech problems. Higher scores were obtained for symptoms classically considered to be late side effects of RT (dental problems, limited mouth opening, dry mouth, sticky saliva). Although teeth problems and trismus could also be due to the oncologic surgery, this observation highlights the long-term persistence of RT-related side effects. Similarly, in a prospective cross-sectional survey on 212 head and neck cancer survivors 1 year after completion of treatment, Chaukar et al. found that dry mouth, sticky saliva, dental problems and mouth opening showed the highest scores [12]. 


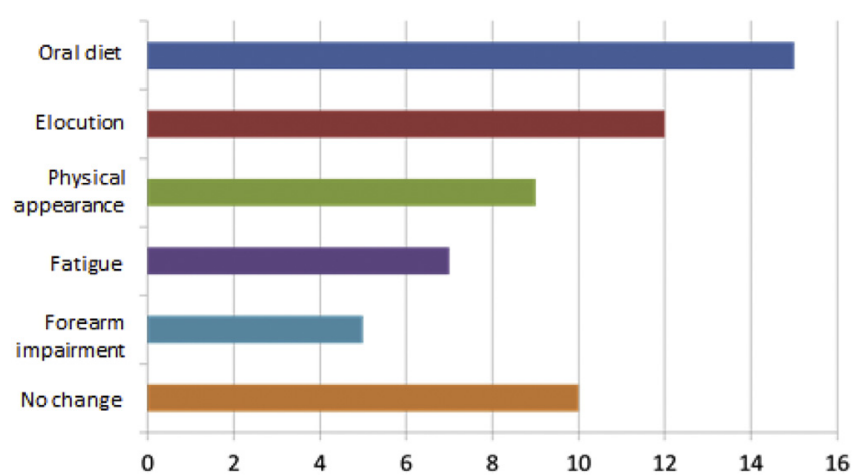

Fig. 1. Patient responses to the open question: "What are the main physical changes since OPC surgery that impact your daily life?"

These problems all contribute to restricted oral feeding long-term after therapy. Furthermore, altered sense of taste, restricted diet and changed physical appearance can all bother the patient when eating in public. This explains why, in our study, as in most published QoL studies in head and neck oncology, post-therapy social eating deteriorated more than swallowing function [8-12].

In the present study, patient speech outcomes seemed satisfactory, with more than $75 \%$ of patients showing a VHI-10 score inferior to 20 and a mean score of 12.8 . Although related, speech and voice are not the same. However, in practice, speech and voice problems can be easily confused. In fact, voice refers to laryngeal function and sound production, whereas speech includes the ability to produce words, sentences and, ultimately, to communicate [13]. Indeed, we recognize that the use of the VHI-10 questionnaire, which is suitable to assess voice problems in patients with laryngeal disorders, is not perfectly appropriate for evaluation of speech problems in patients with OPC. Interestingly, Arffa et al. elucidated normative values for VHI-10 using this questionnaire in 190 patients without voice complaints [14]. They showed a mean VHI-10 score of 2.83 and concluded that a score $>11$ should be considered abnormal. In a recent study evaluating long-term functional outcomes after oncologic surgery and microvascular reconstruction in 64 patients with oral or oropharyngeal cancer, Pierre et al. reported a mean VHI-10 score of 11.2, quite close to our own result, thus demonstrating that speech problems are limited and relatively well-tolerated after this type of surgery [15]. This finding is corroborated by EORTC QLQ-H\&N35 scores showing that speech problems were present but at a lower level than swallowing, eating, and dental or salivary anomalies. In response to the open question: "What are the main physical changes since OPC surgery that impact your daily life?", speech problems were second after changes of oral diet, suggesting that, although pronunciation difficulties are not patients' main complaint, they remain a persistent concern for

Table 5

Predictive factors of clinical outcomes in univariate and multivariate analysis.

\begin{tabular}{|c|c|c|c|c|c|c|c|c|c|c|c|c|}
\hline \multirow[t]{2}{*}{ Variables } & \multicolumn{12}{|c|}{ P values for each factor in univariate/multivariate analyses } \\
\hline & G. QoL & Phys. F. & Role F. & Emot. F. & Cogn. F. & Soc. F. & Gen. S. & H\&N. S. & VHI & DOSS & HADS-A & HADS-D \\
\hline Gender & 0.18 & 0.88 & 0.93 & 0.25 & 0.35 & 0.22 & 0.63 & 0.24 & 0.24 & $\begin{array}{l}0.09 / \\
.46\end{array}$ & 0.16 & $0.08 / .13$ \\
\hline Age $>70$ years & 0.43 & 0.65 & 0.96 & 0.54 & 0.44 & 0.25 & 0.67 & 0.64 & $0.06 / .08$ & 0.35 & 0.76 & 0.25 \\
\hline Low education level & 0.32 & 0.13 & $\underline{0.04 / .07}$ & 0.81 & 0.14 & 0.56 & 0.25 & 0.34 & 0.72 & 0.84 & 0.32 & $0.07 / .10$ \\
\hline $\begin{array}{l}\text { No professional } \\
\text { activity BT }\end{array}$ & 0.27 & $\underline{0.05 / .02}$ & $\overline{0.65}$ & 0.47 & 0.63 & 0.25 & 0.53 & $0.08 / .17$ & 0.57 & 0.60 & 0.99 & 0.64 \\
\hline T-stage $\geq 3$ & 0.79 & 0.58 & 0.97 & 0.32 & 0.98 & 0.19 & 0.51 & 0.58 & 0.34 & 0.89 & $\underline{0.03 / .006}$ & 0.40 \\
\hline N-stage $\geq 1$ & 0.96 & 0.48 & 0.73 & 0.57 & 0.42 & 0.77 & 0.79 & 0.17 & 0.47 & 0.11 & 0.72 & 0.61 \\
\hline Salvage surgery & 0.76 & 0.81 & 0.21 & 0.61 & 0.25 & 0.73 & 0.27 & 0.35 & 0.32 & 0.98 & 0.68 & 0.77 \\
\hline $\begin{array}{l}\text { Base of tongue } \\
\text { involvement }\end{array}$ & 0.66 & 0.27 & 0.73 & 0.98 & 0.88 & 0.41 & 0.85 & 0.89 & 0.89 & 0.67 & 0.20 & 0.69 \\
\hline LPW involvement & $\underline{0.01 / .04}$ & $0.04 / .23$ & 0.16 & $0.09 / .13$ & 0.40 & $\underline{0.03 / .10}$ & $0.09 / .34$ & $\underline{0.006 / .006}$ & $0.08 / .63$ & 0.46 & 0.35 & 0.13 \\
\hline PPW involvement & 0.28 & $\overline{0.67}$ & 0.62 & 0.93 & 0.84 & $\overline{0.07} / .003$ & 0.87 & $\overline{0.11}$ & 0.82 & 0.83 & 0.53 & 0.41 \\
\hline $\begin{array}{l}\text { Soft palate } \\
\text { involvement }\end{array}$ & $\underline{0.009 / .01}$ & 0.72 & 0.20 & $0.07 / \underline{.03}$ & 0.12 & 0.11 & $0.06 / .06$ & 0.41 & 0.82 & 0.91 & 0.74 & 0.74 \\
\hline Transoral approach & 0.20 & 0.24 & 0.93 & 0.80 & 0.60 & 0.52 & 0.44 & $\underline{0.02} / .23$ & $0.09 / .28$ & 0.54 & 0.49 & 0.33 \\
\hline $\begin{array}{l}\text { Transmandibular } \\
\text { approach }\end{array}$ & 0.34 & 0.58 & 0.33 & 0.57 & 0.21 & 0.69 & 0.58 & $\overline{0.74}$ & 0.55 & 0.14 & 0.95 & 0.71 \\
\hline Postoperative $\mathrm{RT} \pm \mathrm{CT}$ & 0.60 & 0.94 & 0.47 & 0.93 & 0.19 & 0.84 & 0.84 & 0.12 & 0.55 & $\begin{array}{l}0.09 / \\
.07\end{array}$ & 0.60 & 0.87 \\
\hline $\begin{array}{l}\text { Alcohol consumption } \\
\text { BT }\end{array}$ & 0.24 & 0.13 & $\underline{0.08 / .13}$ & $\underline{0.05 / .69}$ & 0.17 & $\underline{0.02 / .005}$ & $\underline{0.002 / .02}$ & $\underline{0.001 / .03}$ & 0.13 & 0.54 & $\underline{0.03 / .42}$ & $\underline{0.02 / .01}$ \\
\hline $\begin{array}{l}\text { Alcohol consumption } \\
\text { AT }\end{array}$ & 0.24 & 0.18 & 0.19 & $0.07 / .002$ & 0.63 & $\underline{0.04 / .81}$ & $\underline{0.02 / .81}$ & 0.21 & 0.72 & 0.70 & $0.08 / .45$ & 0.12 \\
\hline $\begin{array}{l}\text { Tobacco consumption } \\
\text { BT }\end{array}$ & 0.74 & 0.12 & 0.61 & $0.07 / .48$ & $\underline{0.02} / .28$ & 0.42 & 0.11 & $\underline{0.02 / .37}$ & 0.93 & $\begin{array}{l}0.09 / \\
.59\end{array}$ & $\underline{0.007 / .04}$ & $\underline{0.05} / .79$ \\
\hline $\begin{array}{l}\text { Tobacco consumption } \\
\text { AT }\end{array}$ & $\underline{0.04 / .21}$ & $\underline{0.02 / .22}$ & 0.17 & 0.11 & $\underline{0.03} / .80$ & $\underline{0.05} / .45$ & $\underline{0.04} / .97$ & 0.33 & 0.44 & 0.32 & $\underline{0.004 / .48}$ & $\frac{<0.001 /}{.009}$ \\
\hline HADS-A & $<\underline{0.001 /-}$ & $<\underline{0.001 /-}$ & $0.001 /-$ & $\leq 0.001 /-$ & $\leq 0.001 /-$ & $\leq 0.001 /-$ & $\leq 0.001 /-$ & $<\underline{0.001 /-}$ & 0.09 & 0.20 & - & $\begin{array}{l}<0.001 / \\
.001\end{array}$ \\
\hline HADS-D & $<\underline{0.001 /-}$ & $<\underline{0.001 /-}$ & $<\underline{0.001 /-}$ & $<\underline{0.001 /-}$ & $<\underline{0.001 /-}$ & $<\underline{0.001 /-}$ & $<\underline{0.001 /-}$ & $<\underline{0.001 /-}$ & $<\underline{0.001 /-}$ & $\underline{0.01 /-}$ & $<\frac{0.001 /}{<0.001}$ & $\overline{-}$ \\
\hline HADS global score & $<\underline{<0.001 /}$ & $<\underline{<0.001 /}$ & $<\underline{<0.001 /}$ & $<\underline{<0.001 /}$ & $<\underline{<0.001 /}$ & $<\underline{<0.001 /}$ & $<\underline{0.001 /}$ & $<\underline{<0.001 /}$ & $\frac{0.008 /}{0.01}$ & $\frac{0.04 /}{.04}$ & $\overline{-}$ & - \\
\hline
\end{tabular}

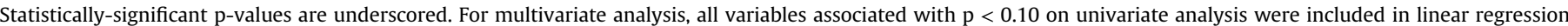

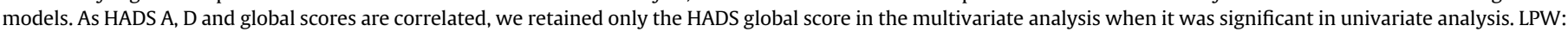

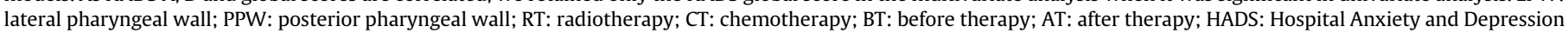

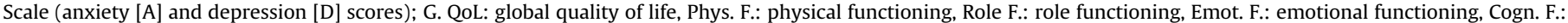

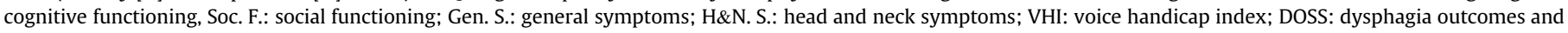
severity scale. 
them long after surgery. Intensive speech therapy in the postoperative period could probably help relieve these problems.

With a mean DOSS score of $5.5(1-7)$ and only $2 \%$ of the patients requiring definitive enteral nutrition (DOSS score $\leq 2$ ), our results regarding swallowing function are very encouraging. More than half the patients recovered normal swallowing and resumed a nonrestricted oral diet. The remaining patients (46\%) had a moderately impaired swallowing function requiring a modified oral diet. These results are corroborated by the mean DHI score (37.5/120) obtained by the patients after therapy, indicating a mild to moderate level of dysphagia. This accounts for a modified oral diet being patients' main complaint in daily life. In a prospective study evaluating swallowing outcomes after intensity-modulated RT for OPC, Guo et al. found a feeding tube dependence rate of $8.3 \%$ one year after therapy [16]. In a systematic review of functional outcomes after transoral robotic surgery, Hutcheson et al. showed that long-term gastrostomy tube rates ranging from $0 \%$ to $7 \%$, were reported by 12 studies in a total of 441 patients, and a mean follow-up in most studies between 1 and 2 years [17]. Taken together, these results demonstrate that, with appropriate microvascular reconstruction, open surgery for OPC provides acceptable long-term swallowing outcomes comparable to those reported with other modern OPC therapeutic strategies.

In the present study, there was no modification in patients' marital status after therapy and most patients reported no change in their family life. The main complaint reported by patients regarding their family life was the decrease in the frequency of sexual intercourse. This is corroborated by the EORTC QLQ-H\&N35 scores showing that a reduced sex life was one of the items with the highest scores. On the other hand, it is also interesting to note that, for some patients, HNSCC and associated treatment resulted in a closer family relationship. In this regard, a recent study on 262 patients with OPC by Taberna et al. showed that relationship distress was infrequently reported, and that $69 \%$ of patients felt that their relationship had strengthened since their cancer was diagnosed [18]. However, the authors also highlighted significant declines in the frequency of vaginal and oral sexual activities at follow-up, regardless of tumor HPV status (abstinence from vaginal and oral sex increased from $10 \%$ to $34 \%$, and from $25 \%$ to $80 \%$, respectively).

Regarding social life, a majority of patients included in the present study reported no change between the pre- and posttherapeutic periods. However, a small proportion of patients reported fewer social contacts and less frequent outings (to restaurants in particular). This relatively good preservation of social life, corroborated by the EORTC QLQ-C30 score superior to 75\% for social functioning, should be emphasized considering the welldocumented social problems encountered by HNSCC patients. In this regard, Veldhuis et al., in a study focusing on QoL in patients with either laryngeal or OPC treated with primary surgery \pm RT, showed that the main complaint of patients with OPC was related to oral functions (xerostomia, mouth opening, sticky saliva) while demonstrating excellent social functioning [19]. In contrast, the authors found that laryngeal cancer patients suffered mainly from coughing and from sensorial and speech problems, and reported more social limitations.

There are very few published data on resumption of professional activity after head and neck cancer treatment. Thus, the present study provides important results in this field of interest. First, we showed that only a small proportion (approximately one third) of patients was still working at the time of surgery. This can be explained by the mean age of OPC diagnosis which is close to the average retirement age in France (around 62 years old). Unlike several western countries where most OPC are related to HPV and where the mean age of patients at diagnosis has gradually declined, in France, most OPC (approximately 70\%) are still imputable to alcohol and tobacco consumption with a mean age at diagnosis virtually unchanged at 60-65 years [2]. Moreover, we showed that approximately half the patients still working at the time of surgery had resumed professional activity at the time of our study.

Two main reasons explain the non-return to work of the other patients. Firstly, due to advanced age, some patients had taken early retirement. Secondly, on account of functional disorders induced by the OPC treatment, some patients had been granted an invalidity pension. In a study assessing employment and return to work in HNSCC survivors conducted in The Netherlands by Verdonck - de Leeuw et al., the authors reported that $83 \%$ of the patients who were employed at the time of diagnosis returned to work after a median period of 6 months [20]. This higher proportion of patients returning to work after treatment and the shorter duration of sick leave compared to our study could be due to the inclusion of all HNSCC survivors, including patients with early-stage disease. In contrast, all the patients included in our study had undergone oncologic surgery with free-flap reconstruction and more than $75 \%$ of them had received multimodal therapy with postoperative $\mathrm{RT} \pm \mathrm{CT}$. Interestingly, Verdonck - de Leeuw et al. also demonstrated that anxiety, oral dysfunction, appetite loss and problems with social eating and social contacts were significantly associated with non-return to work after treatment [20]. In another recent study conducted in India on HNSCC survivors under 60 years, Agarwal et al. showed that $81 \%$ of the patients resumed work and that family structure and level of education were correlated with return to work [21]. Taken together, these results suggest that a multidisciplinary approach including psychosocial supportive interventions is necessary to support and improve return to work among posttreatment HNSCC patients.

Psychological distress is common, even long-term after therapy, in cancer patients, and particularly in those with HNSCC $[22,23]$. Swallowing and speech problems, modified physical appearance, limited social contacts, tobacco and alcohol dependence, as well as fear of recurrence and death, can all contribute to these psychological difficulties. In the present study, based on HADS scores, we found that 26 and $17 \%$ of the patients exhibited an anxious or depressive state, respectively, and that $36 \%$ of the patients suffered from an anxiodepressive disorder. We may assume that part of this anxiodepressive state is attributable to baseline patient psychological characteristics. Indeed, as shown in Table 4, HADS scores and, particularly, the anxiety subscale were already altered before therapy. However, the score obtained before therapy does not reflect the true baseline (i.e. before cancer diagnosis) psychological characteristics of the patients because, before therapy, patients' emotional and psychological status is already affected by cancer diagnosis and the announcement of the therapeutic program. Interestingly, among functioning scales, emotional functioning was the most altered function long-term after therapy. In a recent study on Japanese patients with HNSCC who had undergone surgery, Suzuki et al. found that more than $25 \%$ of patients displayed anxiety or depression, and that higher levels of perceived social support were associated with lower rates of anxiety and depression [24]. In another interesting study on HNSCC patients, Krebber at al. found that psychological distress was present in $29 \%$ of patients and that $82 \%$ of patients identified with an increased level of distress had not yet received psychological support [25]. In a recent prospective randomized study, the same authors showed that stepped care targeting psychological distress was effective and speeded up recovery among HNSCC patients with untreated psychological distress [26]. Altogether, these results suggest that personalized psychosocial supportive interventions are needed in a significant proportion of HNSCC patients even long after treatment and that this type of measure could reduce psychosocial distress among 
patients and ultimately improve their QoL.

As shown in Table 5, alcohol/tobacco consumption before or after therapy were significantly associated with long-term psychological distress. Similarly, regarding QoL scores, we found that alcohol consumption after therapy was an independent predictor of poor emotional functioning. This observation highlights the vicious circle linking psychological distress and alcohol/tobacco addiction, with the former favoring the latter, which in turn reinforces the former. Furthermore, it emphasizes the need for psychological support to help HNSCC patients quit alcohol/tobacco consumption. Although the impact of patients' addictive behaviors (pre-treatment alcohol consumption in particular) on social functioning might appear foreseeable, interestingly, we showed that these addictions also impacted several other QoL scores, such as general and head and neck symptoms. Our study demonstrated that most OPC patients with pre-treatment alcohol or tobacco addiction were able to give up these habits after treatment (more than $60 \%$ for alcohol and over $70 \%$ for smoking). These encouraging results should motivate health-care professionals to evaluate alcohol/tobacco addiction at each post-therapeutic follow-up visit and to adopt a multidisciplinary approach designed to assist HNSCC patients to cease alcohol/tobacco consumption.

In the present study, we found that clinical outcomes had few correlations with tumor stage or site and were not associated with treatment modalities (surgical approach, postoperative treatment). These surprising results could be explained, at least in part, by the homogeneous characteristics of the study cohort, which comprised only OPC patients who had undergone oncologic surgery with RFFF reconstruction. Indeed, most patients had a locally-advanced disease (overall stage III or IV) and had received postoperative RT \pm CT. On the other hand, psychological distress (HADS scores) was an independent predictor of all QoL scores as well as of VHI and DOSS scores, thus highlighting the fact that QoL is more dependent on psychological, emotional and social conditions than on tumor or treatment characteristics. This point must be kept in mind when interpreting the results of QoL studies, particularly in the HNSCC field. Indeed, in a study on 68 patients with oral cancer, Hassanein et al. showed the significant influence of patients' psychological status on post-treatment QoL and reported a significant association between depression and poor functional outcome [27]. In these patients, depression engendered adverse psychological reactions such as fatalism, anxiety and the feeling of powerlessness against the disease. These psychological states were unrelated to gender, age, tumor stage, tumor site or treatment modalities [27]. The above results are corroborated in the study by Ronis et al. on 316 patients with HNSCC which showed that, 1 year post-treatment, the main predictors of QoL, treatment-related factors excepted, were smoking and symptoms of depression [28]. In all, these results suggest that reduction of late therapeutic side-effects and functional rehabilitation is not sufficient to improve long-term QoL in patients with OPC and that a multidisciplinary approach targeting cessation of addictive behaviors and enhancement of psychosocial well-being is also necessary.

The present study has several limitations. First, the number of patients included is relatively low for a study conducted in 4 tertiary care centers. However, these centers are intermediate-volume surgical institutions and the patients included in our study constitute a carefully selected cohort among all OPC patients. A potential bias is that a significant proportion of patients who met the inclusion criteria were probably not enrolled in the study because they were lost to follow-up or because of their refusal to participate. The proportion of such patients is very difficult to evaluate in a study of this kind. As mentioned above, voice is a laryngeal function which is, contrary to speech, not affected significantly in patients with OPC. Thus, we recognize that speech- specific questionnaires like the SHI (speech handicap index) would have been more appropriate than the VHI-10 to evaluate functional outcomes in OPC patients. Another weakness of our study is that we did not conduct a prospective evaluation of QoL from baseline to a clearly-defined period of evaluation. The post-therapeutic evaluation was conducted at long-term (more than 1 year) but at a variable time after treatment. However, as mentioned above, we know that in disease-free cancer patients, QoL does not evolve significantly after 1 year in the absence of other intercurrent disease $[8,10,11]$. Moreover, it was not in the scope of this study to analyze in detail the evolution of QoL and functional results between the pre- and post-therapeutic periods but to focus on longterm clinical outcomes. Nonetheless, our study provides one of the most comprehensive evaluations of long-term QoL and psychosocial well-being and of their predictive factors in OPC survivors who had undergone oncologic surgery with RFFF reconstruction.

\section{Conclusion}

In OPC patients treated by surgery with RFFF reconstruction, long-term QoL and general functions are well-preserved. Main persistent symptoms are fatigue, reduced sexual activity and oral function-related disorders (swallowing, dental, salivary and mouth-opening problems). Approximately half the patients who were still working at the time of diagnosis returned to work. Moreover, there were no major changes in family or social life for most patients, although more than one third of patients developed an anxiodepressive disorder. This psychological distress, along with alcohol/tobacco addiction, are the main determinants of long-term QoL and are therefore critical factors to be considered in the multidisciplinary management of OPC patients.

\section{Conflicts of interest}

We have no conflict of interest to declare.

\section{Appendix A. Supplementary data}

Supplementary data related to this article can be found at https://doi.org/10.1016/j.suronc.2017.11.005.

\section{References}

[1] M. Haigentz Jr., C.E. Silver, J. Corry, et al., Current trends in initial management of oropharyngeal cancer: the declining use of open surgery, Eur. Arch. Otorhinolaryngol. 266 (2009) 1845-1855.

[2] M. Taberna, M. Mena, M.A. Pavón, L. Alemany, M.L. Gillison, R. Mesía, Human papillomavirus related oropharyngeal cancer, Ann. Oncol. 28 (2017) 2386-2398.

[3] M. Roux, O. Dassonville, M. Ettaiche, G. Poissonnet, A. Sudaka, A. Bozec Transoral-transcervical oropharyngectomy without mandibulotomy, associated to fasciocutaneous radial forearm free-flap reconstruction, for oropharyngeal cancer: postoperative course, oncologic and functional results, and prognostic factors, Eur. Ann. Otorhinolaryngol. Head. Neck Dis. 134 (2017) $71-76$.

[4] A. Bozec, G. Poissonnet, E. Chamorey, et al., Quality of life after oral and oropharyngeal reconstruction with a radial forearm free flap: prospective study, J. Otolaryngol. Head. Neck Surg. 38 (2009) 401-408.

[5] S. Al-Hayder, J.J. Elberg, B. Charabi, Clinical outcome and health-related quality-of-life following microsurgical reconstruction in patients with oral and oropharyngeal cancer, Acta Otolaryngol. 137 (2017) 541-545.

[6] S. Høxbroe Michaelsen, C. Grønhøj, J. Høxbroe Michaelsen, et al., Quality of life in survivors of oropharyngeal cancer: a systematic review and meta-analysis of 1366 patients, Eur. J. Cancer 78 (2017) 91-102.

[7] M. Schorr, L.E. Carlson, H.Y. Lau, et al., Distress levels in patients with oropharyngeal vs. non-oropharyngeal squamous cell carcinomas of the head and neck over 1 year after diagnosis: a retrospective cohort study, Support Care Cancer 25 (2017) 3225-3233.

[8] A. Bozec, G. Poissonnet, E. Chamorey, et al., Free-flap head and neck reconstruction and quality of life: a 2-year prospective study, Laryngoscope 118 (2008) 874-880. 
[9] A. de Graeff, J.R. de Leeuw, W.J. Ros, et al., Sociodemographic factors and quality of life as prognostic indicators in head and neck cancer, Eur. J. Cancer 37 (2001) 332-339.

[10] S.N. Rogers, D. Lowe, J.S. Brown, E.D. Vaughan, The University of Washington head and neck cancer measure as a predictor of outcome following primary surgery for oral cancer, Head. Neck 21 (1999) 394-401.

[11] H. Schliephake, M.U. Jamil, Prospective evaluation of quality of life after oncologic surgery for oral cancer, Int. J. Oral Maxillofac. Surg. 31 (2002) 427-433.

[12] D.A. Chaukar, R.R. Walvekar, A.K. Das, et al., Quality of life in head and neck cancer survivors: a cross-sectional survey, Am. J. Otolaryngol. 30 (2009) $176-180$.

[13] R.C. Dwivedi, R.A. Kazi, N. Agrawal, et al., Evaluation of speech outcomes following treatment of oral and oropharyngeal cancers, Cancer Treat. Rev. 35 (2009) 417-424.

[14] R.E. Arffa, P. Krishna, J. Gartner-Schmidt, C.A. Rosen, Normative values for the voice handicap Index-10, J. Voice 26 (2012) 462-465.

[15] C.S. Pierre, O. Dassonville, E. Chamorey, et al., Long-term functional outcomes and quality of life after oncologic surgery and microvascular reconstruction in patients with oral or oropharyngeal cancer, Acta Otolaryngol. 134 (2014) 1086-1093.

[16] G.Z. Guo, K.R. Sutherland, C. Myers, P. Lambert, S.K. Loewen, H.C. Quon, Prospective swallowing outcomes after IMRT for oropharyngeal cancer: dosimetric correlations in a population-based cohort, Oral Oncol. 61 (2016) $135-141$.

[17] K.A. Hutcheson, F.C. Holsinger, M.E. Kupferman, J.S. Lewin, Functional outcomes after TORS for oropharyngeal cancer: a systematic review, Eur. Arch. Otorhinolaryngol. 272 (2015) 463-471.

[18] M. Taberna, R.C. Inglehart, R.K. Pickard, C. Fakhry, A. Agrawal, M.L. Katz, M.L. Gillison, Significant changes in sexual behavior after a diagnosis of human papillomavirus-positive and human papillomavirus-negative oral cancer, Cancer 123 (2017) 1156-1165.

[19] D. Veldhuis, G. Probst, A. Marek, et al., Tumor site and disease stage as predictors of quality of life in head and neck cancer: a prospective study on patients treated with surgery or combined therapy with surgery and radiotherapy or radiochemotherapy, Eur. Arch. Otorhinolaryngol. 273 (2016) 215-224.

[20] I.M. Verdonck-de Leeuw, W.J. van Bleek, C.R. Leemans, R. de Bree, Employment and return to work in head and neck cancer survivors, Oral Oncol. 46 (2010) 56-60.

[21] J. Agarwal, R. Krishnatry, P. Chaturvedi, et al., Survey of return to work of head and neck cancer survivors: a report from a tertiary cancer center in India, Head. Neck 39 (2017) 893-899.

[22] R.L. Holloway, J.L. Hellewell, A.M. Marbella, P.M. Layde, K.B. Myers, B.H. Campbell, Psychosocial effects in long-term head and neck cancer survivors, Head. Neck 27 (2005) 281-288.

[23] B. Barber, J. Dergousoff, M. Nesbitt, et al., Depression as a predictor of postoperative functional performance status (PFPS) and treatment adherence in head and neck cancer patients: a prospective study, J. Otolaryngol. Head. Neck Surg. 44 (2015) 38.

[24] M. Suzuki, M. Deno, M. Myers, et al., Anxiety and depression in patients after surgery for head and neck cancer in Japan, Palliat. Support Care 14 (3) (2016 269-277.

[25] A.M. Krebber, F. Jansen, P. Cuijpers, C.R. Leemans, I.M. Verdonck-de Leeuw Screening for psychological distress in follow-up care to identify head and neck cancer patients with untreated distress, Support Care Cancer 24 (2016) 2541-2548.

[26] A.M. Krebber, F. Jansen, B.I. Witte, et al., Stepped care targeting psychologica distress in head and neck cancer and lung cancer patients: a randomized, controlled trial, Ann. Oncol. 27 (2016) 1754-1760.

[27] K.A. Hassanein, B.T. Musgrove, E. Bradbury, Psychological outcome of patients following treatment of oral cancer and its relation with functional status and coping mechanisms, J. Craniomaxillofac Surg. 33 (2005) 404-409.

[28] D.L. Ronis, S.A. Duffy, K.E. Fowler, M.J. Khan, J.E. Terrell, Changes in quality of life over 1 year in patients with head and neck cancer, Arch. Otolaryngol. Head. Neck Surg. 134 (2008) 241-248. 